\title{
Escala de Creencias sobre la Maternidad: Construcción y Validación en Mujeres Mexicanas
}

\author{
Maternity Beliefs Scale: Construction and Validation in Mexican Women
}

\author{
Cecilia Mota González ${ }^{1}$, Nazira Calleja ${ }^{2}$, Claudia Sánchez Bravo ${ }^{3}$ y Jorge Carreño Meléndez ${ }^{4}$
}

\begin{abstract}
Resumen
La maternidad se ha transformado a lo largo de la historia. Actualmente, está matizada por la igualdad de género, los avances tecnológicos y los avances científicos, por lo que surge el interés de construir y determinar las características psicométricas de una Escala de Creencias sobre la Maternidad. Fue aplicada a 545 mujeres mexicanas con diferente estatus materno (con pérdida perinatal, infértiles, con embarazo de alto riesgo, madres de niños en edad preescolar y estudiantes universitarias sin hijos). La escala quedó conformada finalmente por 13 reactivos, agrupados en dos subescalas: maternidad como sentido de vida y maternidad como deber social. Los índices de confiabilidad y validez resultaron satisfactorios. Se discute su utilidad en investigación y en la clínica, y se analizan los resultados en términos de conceptualización de la maternidad como una elección que complementa la vida femenina y no como un deber.
\end{abstract}

Palabras clave: maternidad, creencias, escalas psicométricas, rol de género, actitudes

\begin{abstract}
Motherhood has been transformed throughout history. Nowadays, it is also nuanced by gender equity and both technological and scientific progress, hence the interest in establishing and determining the psychometric characteristics of a Maternity Beliefs Scale. The scale was applied to 545 Mexican women with different maternal statuses (perinatal loss, infertility, high-risk pregnancy, mothers with preschool children and university students without children). The scale is constituted by 13 test items, arranged in two subscales: motherhood as a sense of life and motherhood as a social duty. Both indexes for reliability and validity were satisfactory. Discussion includes their usefulness in research and clinical practice, and the results are analyzed in terms of the conceptualization of motherhood as a complementary choice for the life of a woman and not as a duty.
\end{abstract}

Keywords: maternity, beliefs, psychometric scales, gender role, attitudes

\footnotetext{
${ }^{1}$ Maestra en Psicología. Instituto Nacional de Perinatología. Investigador en Ciencias Médicas. Montes Urales 800, Lomas Virreyes CP.11000, Ciudad de México, México. Tel.: 55209900 ext. 147. Correo: motaceci@ hotmail.com

2 Doctora en Psicología. Universidad Nacional Autónoma de México. Investigador Titular B, Facultad de Psicología. Avenida Universidad 3000, CP. 04510, Ciudad Universitaria, Ciudad de México, México. Tel.: 52551126-6797. Correo: ncalleja@unam.mx

${ }^{3}$ Doctora en Psicología. Instituto Nacional de Perinatología. Coordinadora del Departamento de Psicología. Montes Urales 800, Lomas Virreyes CP.11000, Ciudad de México, México. Tel.: 55209900 ext. 147. Correo: clausanbra@ yahoo.com

${ }^{4}$ Doctor en Psicología. Instituto Nacional de Perinatología. Investigador en Ciencias Médicas. Montes Urales 800, Lomas Virreyes CP.11000, Ciudad de México, México. Tel.: 55209900 ext. 147. Correo: jocame2003@yahoo.com
} 


\section{Introducción}

A lo largo de la historia, las creencias sobre la maternidad han sufrido cambios, influidos por factores culturales y sociales. En la cultura latina, la maternidad es significativa para mujeres y hombres y ha sido un elemento poderoso para la autodefinición y autoevaluación de la mujer (Contreras \& Reyes, 2007; Molina, 2006). Del siglo XVIII a la primera mitad del XX, la maternidad se consideró el rol central de la mujer. La identidad femenina tradicional la colocaba como el eje de articulación de la femineidad, de manera tal que la historia personal, las decisiones y el proyecto de vida de la mayoría de las mujeres se estructuraba en torno a ser madre (Mota, Aldana, \& Gómez 2013), que implicaba ser guardiana y responsable tanto de la salud física y moral de sus hijos como de su bienestar psicológico. La maternidad era un ideal por ser una función noble, gratificante y edificante.

La sociedad contemporánea ha dejado atrás esta postura. Ahora, los conceptos de maternidad están inmersos en la igualdad de género, los avances tecnológicos y el progreso científico e influidos por los cambios en los patrones demográficos, sexuales y reproductivos, que han llevado a las mujeres a disminuir el número de hijos y a incrementar su participación en la vida pública (Ehrenberg, 2000).

Actualmente, es posible que las mujeres cuestionen si la maternidad es esencial para su identidad (Mendoza, 2012). La relevancia que esta tiene para su vida difiere según su grupo social, su cultura y sus creencias, de manera que cuanto mayor es su formación académica y su inserción en una actividad laboral, sus posibilidades de realización personal se diversifican (Agudelo, Bedoya, \& Osorio, 2016; Colondro, Limiñana, \& Ordoñana, 2014). Un estudio realizado con mujeres polacas que decidieron no tener hijos y desarrollarse profesional y laboralmente, mostró que su elección se basó en decisiones pragmáticas relacionadas con el entorno político, laboral y de género (Mishtal, 2012). Álvarez (2013) encontró que la decisión de mujeres españolas de tener hijos (y de cuándo tenerlos) dependió, entre otros factores, de su edad y de su estabilidad económica y laboral. Otras investigaciones también subrayan que las mujeres en la actualidad han experimentado cambios fundamentales en su identidad y conciben a la maternidad como una circunstancia difícil de combinar con otras aspiraciones, ya que requiere tiempo completo (Ávila, 2005; Maher \& Saugeres, 2007; Mitchell \& Gray, 2007).

Sin embargo, en América Latina el paradigma dominante sigue siendo "nacer mujer es nacer para ser madre", es decir, supone que el destino de la vida femenina es la maternidad. Valladares (1997) señala que, a través de la idea "mujer=madre", las sociedades organizan el mito de la maternidad y con él las prescripciones que guían los diferentes comportamientos en el concebir, parir y criar a los hijos. Romero, Sánchez, Romero y Chávez (2010) afirman que esta es una forma en que las latinas adquieren poder y prestigio social.

Si bien en México existe un discurso genérico sobre la mujer, cada grupo social jerarquiza los significados de la maternidad e interioriza determinadas actitudes y creencias. Arranz, Blum, Sauceda y Gutiérrez (2001) señalan que socialmente se espera que las mexicanas sean afectuosas, tiernas, abnegadas y sobreprotectoras de los hijos, prevaleciendo los mensajes impregnados de normas e ideas morales culpígenas respecto de cómo ser una "buena" madre. No obstante, el mito de la maternidad intensiva no siempre es operativo, pues para muchas mujeres la maternidad ha dejado de ser su única fuente de realización (Mota, Calleja, Gómez, Aldana, \& Sánchez, 2010).

Esta discrepancia entre los modelos "ideales" de maternidad y sus deseos, y la realidad actual de las mujeres, conlleva con frecuencia efectos negativos en su salud física y refuerza la inseguridad y el sentimiento de culpa de muchas de ellas. Tal es el caso de quienes, por circunstancias biológicas o médicas, no logran ser madres. Nazario y Turato (2007) señalan que cuando existe una enfermedad crónica en la que el embarazo está contraindicado, se presenta un deseo más intenso de ser madre, provocado tanto por el deseo de dar continuidad a la vida como por la presión social. Coté y Morrison (2001) compararon las creencias de mujeres que habían tenido abortos con las de aquellas que habían parido un hijo y encontraron que las primeras valoraban más la maternidad que las segundas. 
Otras investigaciones sobre las repercusiones emocionales de no poder ser madre han concluido que quienes han abortado, sufrido una muerte perinatal o son infértiles, ven afectada su identidad, su valor personal y su imagen corporal, además de que el valor que la maternidad tiene para ellas cobra mayor fuerza (Cardenas, Chavez, \& Lozano, 2016; González, Carreño, Sánchez, \& Morales, 2013; Rivero, Quintero, Montiel, Karame, \& Alarcón, 2015;). El reconocimiento de la incapacidad para procrear puede traer consecuencias psicológicas que van más allá de la ausencia de niños o de la función de crianza.

Puesto que la representación de la maternidad varía según la época y los contextos socioculturales, es fundamental conocer las creencias que actualmente tienen las mujeres acerca de la maternidad, para lo cuál son necesarios los instrumentos de medición. Sin embargo, la mayoría de los instrumentos relacionados con la maternidad se enfocan en la eficacia de las mujeres en el cuidado del bebé, por ejemplo la Self Efficacy in Infant Care Scale, (Prasopkittikun, Tilokskulchai, Sinsuksai, \& Sitthimongkol, 2006) o en la satisfacción y los sentimientos de autoeficacia de las madres, como la Escala de Evaluación Parental (Farkas-Klein, 2008), y no en sus creencias hacia la maternidad, que son importantes para entender el comportamiento y el desarrollo de problemas emocionales relacionados con la fertilidad. Adicionalmente, los instrumentos desarrollados en una determinada cultura pueden incluir actividades poco usuales para las mujeres de otros países, o pueden variar en su significación social (Casullo, 2009). Por tanto, el objetivo del presente estudio fue desarrollar y validar un instrumento para medir las creencias sobre la maternidad que tienen las mujeres mexicanas, que pueda ser utilizado tanto en la investigación de temas relacionados a la maternidad como en la intervención psicológica.

Para determinar la validez concurrente de la escala, se incluyeron en el estudio mujeres con diferente estatus materno (con pérdida perinatal, infértiles, con embarazo de alto riesgo, madres de niños en edad preescolar y estudiantes universitarias sin hijos) y con características demográficas diversas.

\section{Método}

\section{Participantes}

Participaron 545 mujeres, con edades entre los 17 y los 52 años (Media=30.97; DT=7.54), que asistían a un hospital especializado en salud reproductiva en la Ciudad de México debido a que habían sufrido: una pérdida perinatal (35.8\%), o estaban en tratamiento por infertilidad, o embarazo de alto riesgo (10.8\%), así como por madres de niños en edad preescolar $(36.6 \%)$ y por estudiantes universitarias sin hijos (17.1\%). La muestra fue no aleatoria, intencional por cuota. Como se observa en la Tabla 1, el $64.7 \%$ tenían una escolaridad media superior o superior, y una tercera parte un trabajo remunerado. Cuatro de cada diez eran solteras y una proporción similar tenía de uno a cinco hijos. La mitad experimentó una o más pérdidas perinatales y el $5 \%$ se encontraban embarazadas (ver Tabla 2).

Tabla 1. Características sociodemográficas de las

\begin{tabular}{lc}
\multicolumn{2}{c}{ participantes } \\
\hline Variable & $\%$ \\
\hline Escolaridad & \\
Primaria & 4.1 \\
Secundaria & 19.1 \\
Preparatoria & 27.5 \\
Universidad & 37.2 \\
Posgrado & 12.1 \\
Total & 100.0 \\
Trabajo Remunerado & \\
No & 61.8 \\
Si & 38.2 \\
Total & 100.0 \\
Estado Civil & \\
Soltera & 41.5 \\
Casada & 39.3 \\
Unión Libre & 14.9 \\
Otro & 4.3 \\
Total & 100.0 \\
\hline
\end{tabular}

Tabla 2. Características reproductivas de las participantes

\begin{tabular}{lc}
\hline Variable & $\%$ \\
\hline Número de Hijos & \\
Sin Hijos & 60.0 \\
1 & 14.1 \\
2 & 17.2 \\
3 ó más & 8.7 \\
Total & 100.0 \\
Pérdida de Hijos & \\
Si & 50.1 \\
No & 49.9 \\
Total & 100.0 \\
Embarazo Actual & \\
Embarazada & 4.8 \\
No Embarazada & 95.2 \\
Total & 100.0 \\
\hline
\end{tabular}




\section{Instrumentos}

\section{Cuestionario sociodemográfico y de variables relativas a los hijos}

Las preguntas del instrumento, en su mayoría cerradas, recabaron información respecto de su edad, escolaridad, trabajo remunerado, estado civil, número de hijos, si habían perdido hijos durante el embarazo o alrededor del nacimiento, $y$ si se encontraban embarazadas en la actualidad.

\section{Escala de Creencias sobre la Maternidad}

Elaboración de los reactivos. A partir de la definición del constructo, se entrevistó a mujeres que asistían a consulta ginecológica a un hospital especializado en salud reproductiva, madres de niños preescolares y estudiantes universitarias. Se pidió que comentaran el significado de ser madre para la mujer. Se grabaron y transcribieron las frases expresadas. El análisis de contenido de éstas mostró dos dimensiones de las creencias sobre la maternidad: como sentido de vida y como deber social.

Las creencias en la maternidad como sentido de vida se referían a consideraciones respecto de que ser madre es lo que da significado o finalidad a la existencia de una mujer, lo que les hace sentirse felices y realizadas; las creencias en la maternidad como deber social señalaban que una mujer tiene la obligación, ante la sociedad, de ser madre; si no lo es, su valor como persona será escaso. Con tales frases se elaboraron 17 reactivos en una sola dirección.

Validación de contenido. Se solicitó a tres jueces que evaluaran la concordancia del contenido de cada reactivo con la definición de la dimensión propuesta, la claridad y la adecuación de su redacción a las normas psicométricas. Dos de los reactivos fueron juzgados como no pertinentes para la medición del constructo (quedando 15), y se propusieron adecuaciones para tres de las frases en términos de su redacción. Se establecieron seis opciones de respuestas tipo Likert, de Totalmente en desacuerdo (0) a Totalmente de acuerdo (5).

\section{Procedimiento}

Ambos instrumentos, el Cuestionario sociodemográfico y ginecológico, y la Escala de Creencias sobre la Maternidad, fueron aplicados de manera piloto a 15 mujeres que asistían a consulta ginecológica en una institución hospitalaria. Se pidió que respondieran los instrumentos y se les preguntó sobre la redacción y claridad de las instrucciones y de los reactivos; asimismo, se solicitó que expresaran dudas o sugerencias sobre ellos. A partir de la aplicación piloto, se modificaron algunas palabras en los reactivos confusos.

La aplicación final se realizó en tres ámbitos diferentes: en un hospital especializado en salud reproductiva, en tres escuelas de niños preescolares y en una universidad pública. En cada caso, la aplicación se efectuó de manera individual.

Respecto de las consideraciones éticas, a cada participante se le explicaron las condiciones de su inclusión en el estudio, el carácter anónimo de su información y su participación voluntaria. La recolección de los datos fue realizada por psicólogos entrenados.

\section{Análisis de los datos}

Los datos se analizaron mediante los programas SPSS y AMOS versión 22. Para el análisis psicométrico de la escala se realizó un análisis factorial exploratorio con el método de máxima verosimilitud y rotación oblicua promax, y un análisis factorial confirmatorio, utilizando los índices de ajuste del modelo $\chi^{2} / \mathrm{gl} ; A G F I ; C F I y$ RMSEA. Se obtuvieron índices alpha de Cronbach de consistencia interna e índices de correlación de Pearson. Para diferencias entre grupos, se aplicaron pruebas $t$ y Anova de un factor.

\section{Resultados}

\section{Validación de constructo}

El análisis factorial exploratorio mostró el valor de adecuación muestral KMO de .943 y el de Esfericidad de Barltett resultó estadísticamente

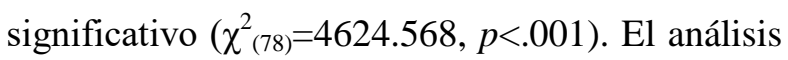
convergió en tres iteraciones arrojando dos factores con valores eigen $>1$, que explicaron el $59.451 \%$ de la varianza total. Los factores obtenidos correspondieron a las dimensiones planteadas teóricamente (ver Tabla 3). Dos reactivos (10. "Un hombre quiere más a su esposa si tiene hijos" y 14. "Las personas admiran a las mujeres que tienen hijos") resultaron con cargas factoriales <.40, por lo que fueron eliminados. $\mathrm{La}$ correlación entre los factores fue alta $(\mathrm{r}=.721)$. 
Tabla 3. Estructura factorial de la Escala de Creencias sobre la Maternidad

\begin{tabular}{lcc}
\hline \multirow{2}{*}{ Reactivo } & \multicolumn{2}{c}{ Maternidad } \\
\cline { 2 - 3 } 12. Lo más importante para una mujer es ser madre & Como sentido de vida & Como deber social \\
3. Lo que más desea una mujer es tener uno o más hijos & .87 & \\
7. Una mujer es más feliz si es madre & .85 & .82 \\
4. Para una mujer, ningún logro se compara con ser madre & .77 & .64 \\
9. Para sentirse feliz, una mujer necesita tener un hijo & .61 & .61 \\
5. Una mujer está completa hasta que es madre & .51 & .94 \\
11. Una mujer se realiza hasta que tiene un hijo. & & .71 \\
2. La vida vale la pena si tienes hijos. & & .71 \\
13. Si una mujer no tiene hijos, merece el rechazo de los demás & .69 \\
15. Para una mujer vale la pena vivir solo si tiene hijos. & .41 \\
1. El valor de una mujer depende de que sea madre & & \\
6. El deber de una mujer es tener hijos & & \\
8. Los hombres respetan más a la mujer cuando es madre & & \\
\hline
\end{tabular}

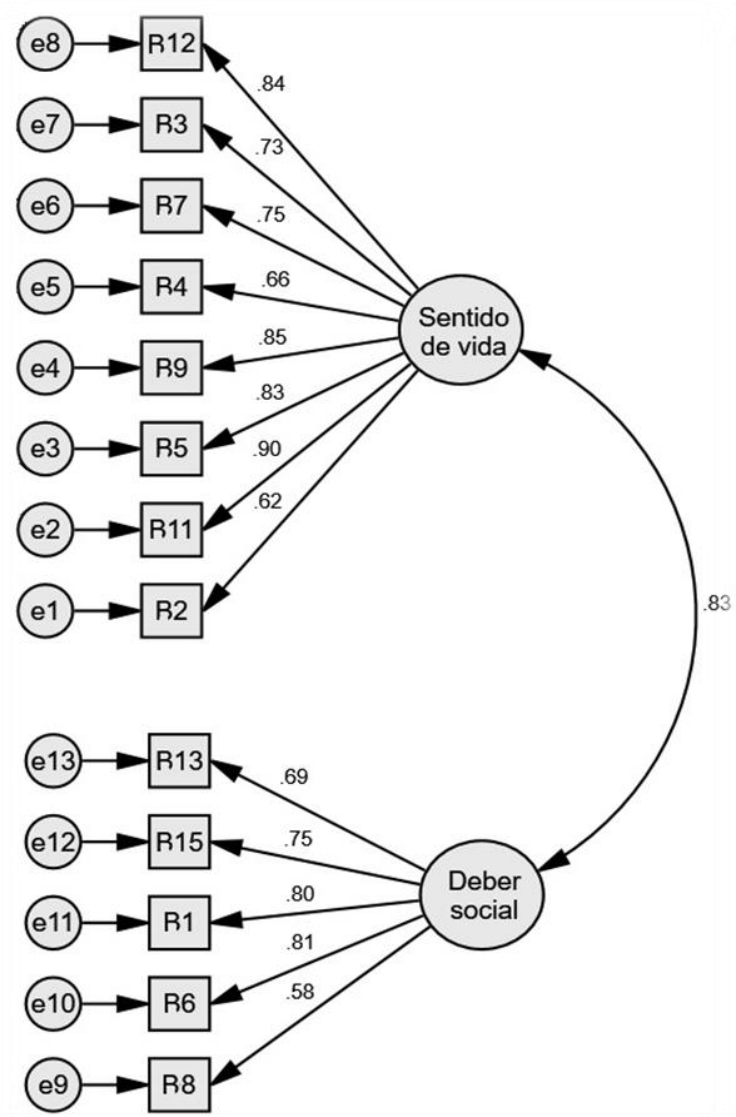

Figura 1. Modelo del análisis factorial confirmatorio de la escala

Con los resultados obtenidos en el análisis factorial exploratorio, se efectuó un análisis factorial confirmatorio. Una vez hechas las modificaciones propuestas para el modelo, los índices obtenidos mostraron un buen ajuste $\left(\chi^{2} / \mathrm{gl}=162.486 / 59=2.754 ; \mathrm{AGFI}=.970 ; \mathrm{CFI}=.962 ;\right.$ RMSEA = .049; IC90\%: .045-.052). Todos los reactivos tuvieron cargas significativas sobre sus respectivos factores, con parámetros estandarizados que oscilaron entre .58 y .90 (Figura 1 ).
Se evaluó la consistencia interna de la escala y de los dos factores resultantes. El índice para la escala total fue $\alpha=.927$; para el factor Maternidad como sentido de vida, $\alpha=.917$; para el factor Maternidad como deber social, $\alpha=.831$

Validación con grupos contrastados.

Para obtener la validez de criterio se compararon los puntajes obtenidos por las cuatro categorías de participantes en términos de su estatus materno: dos clínicas (mujeres con pérdida perinatal y mujeres en tratamiento por infertilidad o embarazo de alto riesgo) y dos no clínicas (madres de niños en edad preescolar y estudiantes universitarias sin hijos). Se esperaba que las mujeres con pérdida perinatal cuyo rol como madres es incierto mostraran puntajes más altos, en tanto que las estudiantes universitarias sin hijos obtendrían puntuaciones más bajas en ambos factores. Los resultados de la prueba Anova de un factor mostrados en la tabla 4 indicaron que, en ambas subescalas, las mujeres con pérdida perinatal puntuaron más alto que las infértiles, y éstas obtuvieron mayores puntajes que las madres de preescolares, quienes, a su vez, puntuaron por arriba de las estudiantes sin hijos. En la subescala de Maternidad como sentido de vida, todas las comparaciones post hoc resultaron significativas, y en la de Maternidad como deber social, las mujeres con pérdida perinatal se diferenciaron significativamente de las madres de preescolares y de las estudiantes, pero no de las mujeres infértiles. El porcentaje de varianza explicada para la primera subescala fue de $35 \%$, y para la segunda, 12\%. En general, los puntajes de las creencias de Maternidad como sentido de vida fueron más altos $(\mathrm{M}=1.69, \mathrm{DT}=1.34),(\mathrm{M}=0.77$, 
Tabla 4. Diferencias de medias entre los diferentes grupos de mujeres en las dos subescalas

\begin{tabular}{|c|c|c|c|c|c|}
\hline Subescala & Grupo & Media (DT) & $\mathrm{F}$ & Tamaño del efecto & $\begin{array}{l}\text { Post hoc } \\
\text { Bonferroni }\end{array}$ \\
\hline \multirow[t]{2}{*}{$\begin{array}{l}\text { Maternidad como } \\
\text { sentido de vida }\end{array}$} & Mper. & $2.68(1.33)$ & \multirow[t]{2}{*}{$\begin{array}{l}\mathrm{F}(3.541)= \\
98.787^{* *}\end{array}$} & \multirow[t]{2}{*}{$\eta^{2}=.35$} & $\begin{array}{l}\text { Mper. - Minf. ** } \\
\text { Mper - Mpre.** } \\
\text { Mper - Est.** }\end{array}$ \\
\hline & $\begin{array}{l}\text { Minf. } \\
\text { Mpre. } \\
\text { Est. }\end{array}$ & $\begin{array}{l}1.79(1.00) \\
1.19(0.99) \\
0.62(0.65)\end{array}$ & & & $\begin{array}{l}\text { Minf - Mpre.** } \\
\text { Minf - Est.** } \\
\text { Mpre -Est.** }\end{array}$ \\
\hline \multirow[t]{2}{*}{$\begin{array}{l}\text { Maternidad como deber } \\
\text { social }\end{array}$} & Mper. & $1.16(1.08)$ & \multirow[t]{2}{*}{$\begin{array}{l}\mathrm{F}(3.541)= \\
24.060 * *\end{array}$} & \multirow[t]{2}{*}{$\eta^{2}=.12$} & $\begin{array}{l}\text { Mper - Minf. ns } \\
\text { Mper - Mpre.** } \\
\text { Mper - Est.** }\end{array}$ \\
\hline & $\begin{array}{l}\text { Minf. } \\
\text { Mpre. } \\
\text { Est. }\end{array}$ & $\begin{array}{l}0.87(0.94) \\
0.52(0.75) \\
0.42(0.48)\end{array}$ & & & $\begin{array}{l}\text { Minf - Mpre* } \\
\text { Minf - Est.** } \\
\text { Mpre -Est. ns }\end{array}$ \\
\hline
\end{tabular}

Nota. Mper=Mujeres con pérdida; Minf=Mujeres infertiles; Mpre=Madres de preescolares; Est=Mujeres estudiantes; $\mathrm{ns}=$ no significativo.

$* p<.005, * * p<.001$

Tabla 5. Diferencias por escolaridad en las dos subescalas de Maternidad

\begin{tabular}{|c|c|c|c|c|c|}
\hline Subescala & Grupo & Media (DT) & $\mathrm{F}$ & Tamaño del efecto & $\begin{array}{l}\text { Pos hoc } \\
\text { Bonferroni }\end{array}$ \\
\hline $\begin{array}{l}\text { Maternidad como } \\
\text { sentido de vida }\end{array}$ & $\begin{array}{l}\text { Pr. } \\
\text { Sec. } \\
\text { Bach. } \\
\text { Uni. } \\
\text { Pos. }\end{array}$ & $\begin{array}{l}3.27(1.15) \\
2.35(1.31) \\
2.03(1.32) \\
1.18(1.14) \\
0.91(0.85)\end{array}$ & $\begin{array}{c}\mathrm{F}(4.540)= \\
52.618^{* *}\end{array}$ & $\eta^{2}=.21$ & $\begin{array}{l}\text { Pr - Sec. }{ }^{*} \\
\operatorname{Pr}-\text { Bach.** } \\
\text { Pr - Uni.** } \\
\text { Pr - Pos.** } \\
\text { Sec-Bach. ns } \\
\text { Sec- Uni. ** } \\
\text { Sec-Pos ** } \\
\text { Bach - Uni ** } \\
\text { Bach - Pos ** } \\
\text { Uni - Pos. ns }\end{array}$ \\
\hline $\begin{array}{l}\text { Maternidad como } \\
\text { deber social. }\end{array}$ & $\begin{array}{l}\text { Pr. } \\
\text { Sec. } \\
\text { Bach. } \\
\text { Uni. } \\
\text { Pos. }\end{array}$ & $\begin{array}{l}2.00(1.35) \\
1.01(1.04) \\
0.88(0.95) \\
0.56(0.71) \\
0.37(0.55)\end{array}$ & $\begin{aligned} & \mathrm{F}(4.540) \\
= & 15.022^{* *}\end{aligned}$ & $\eta^{2}=.13$ & $\begin{array}{l}\operatorname{Pr}-\text { Sec. } * * \\
\operatorname{Pr}-\text { Bach.** } \\
\text { Pr }- \text { Uni.** } \\
\text { Pr }- \text { Pos.** } \\
\text { Sec - Bach. ns } \\
\text { Sec - Uni. ** } \\
\text { Sec - Pos ** } \\
\text { Bach - Uni ** } \\
\text { Bach - Pos ** } \\
\text { Uni - Pos. ns }\end{array}$ \\
\hline
\end{tabular}

Nota. Pr.=Primaria; Sec.=Secundaria; Bach.=Bachillerato; Uni.=Universidad; Pos.=Posgrado; ns=no significativo.

$* p<.005, * * p<.001$.

$\mathrm{DT}=0.92)$ que los de Maternidad como deber social $(\mathrm{M}=0.77$, $\mathrm{DT}=0.92)$; en ambas subescalas las medias estuvieron por debajo de la media teórica (2.5).

\section{Creencias sobre la maternidad y variables} sociodemográficas. Se evaluó la relación existente entre las creencias sobre la maternidad y la edad, la escolaridad, el trabajo remunerado y el número de hijos. Se observó una fuerte correlación positiva entre la edad y las creencias de la maternidad como sentido de vida $(\mathrm{r}=.716$, $p<.001)$, y una relación débil, aunque significativa, con las creencias de la maternidad como deber social ( $\mathrm{r}=.137, p=.001)$; los coeficientes de determinación fueron, respectivamente, $\mathrm{r}^{2}=.51, \mathrm{y}$ $r^{2}=.02$

Se encontraron diferencias altamente significativas en ambas subescalas por escolaridad: quienes sólo tuvieron educación básica (primaria) obtuvieron los puntajes más altos, seguidas por las de estudios de secundaria y de bachillerato o técnico, siendo los más bajos los de las mujeres con estudios superiores (licenciatura y posgrado). Las pruebas de comparaciones múltiples resultaron significativas, 

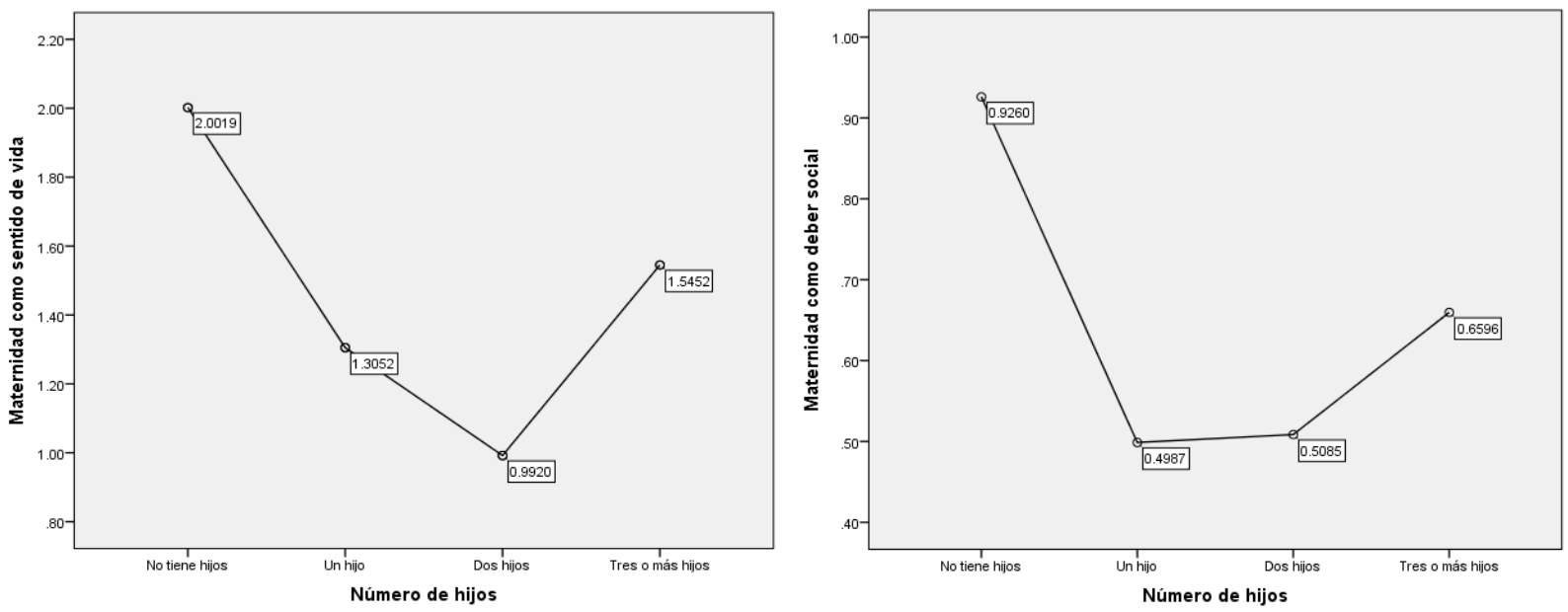

Figura 2. Medias de puntajes en las subescalas de maternidad como sentido de vida y maternidad como deber social

excepto para las de secundaria contrastadas contra las de bachillerato, y las de licenciatura contra las de posgrado. La escolaridad explicó el $21 \%$ de la varianza de los puntajes en la subescala de Maternidad como sentido de vida, y el $13 \%$ en la de Maternidad como deber social (ver Tabla 5).

Las participantes que no trabajaban mostraron puntajes significativamente mayores en las dos subescalas $(\mathrm{M}=1.88 ; \mathrm{DT}=1.42$ para Maternidad como sentido de vida, y $\mathrm{M}=0.86$; $\mathrm{DT}=0.97$ para Maternidad como deber social), comparados con quienes sí trabajaban $(\mathrm{M}=1.39, \quad \mathrm{DT}=1.15 \mathrm{y}$ $\mathrm{M}=0.86, \quad \mathrm{DT}=0.97, \quad$ respectivamente), $\mathrm{t}(542)=4.197, p<.001, \mathrm{~d}=.38$, y $\mathrm{t} \quad(542)=2.728$, $p=.007, \mathrm{~d}=.27$.

Respecto al número de hijos y las creencias sobre la maternidad, se encontró que las mujeres sin hijos y aquellas que eran madres de tres o más, puntuaron significativamente más alto que aquellas que tenían uno o dos hijos, $F$ $(3,541)=18.151, p<.001, \eta^{2}=.09$, para Maternidad como sentido de vida, y F $(3,541)=8.375$, $\mathrm{p}<.001$, $\eta^{2}=.04$ para Maternidad como deber social. Las dos primeras no se diferenciaron entre sí, según las pruebas post hoc de Bonferroni, al igual que las dos últimas (ver Figura 2).

\section{Discusión}

Retomando lo mencionado por Molina (2006), a lo largo de la historia se ha observado que el rol de la maternidad es de gran significado en la vida de las mujeres latinas. Las creencias sobre la maternidad tal como lo señala Valladares (2007), están matizadas por el mito social de "mujer=madre" y constituyen un hecho cultural aprendido más que un proceso biológico e instintivo. La presente investigación aporta la construcción de una escala que evalúa las creencias de las mujeres mexicanas sobre la maternidad, la cual empieza a ser considerada no como la única fuente de realización femenina sino como una parte de su identidad (Mota et.al., 2010).

La escala construida en el presente estudio quedó conformada por 13 reactivos, distribuidos en dos subescalas, que exploran las creencias hacia la maternidad como sentido de vida y como deber social. Esta conformación, obtenida mediante análisis factorial exploratorio, fue corroborada a través del análisis factorial confirmatorio. Los índices de confiabilidad y de validez de criterio de la escala también resultaron satisfactorios. Además, constituye un instrumento de fácil aplicación.

Contar con un instrumento confiable y válido que evalúe las creencias sobre la maternidad resulta de gran utilidad tanto en la investigación como en la práctica clínica.

En concordancia con lo mencionado por Álvarez (2007), en México, como en la mayoría de los países que experimentan cambios vertiginosos, se ha visto afectada la forma de vida de la población de hombres y mujeres. Esto ha impactado el proceso de afrontamiento de la maternidad, por lo que la competencia laboral, la lucha por obtener una posición social, la búsqueda del bienestar y el deseo de lograr una mejor 
calidad de vida, se contraponen con la necesidad biológica y psicológica de la maternidad y con las expectativas socioculturales en torno a ella. Hoy en día, las mujeres que tienen acceso a la educación y al desarrollo de una vida profesional y laboral dan un valor menor a la maternidad, optan por un número menor de hijos y posponen la procreación. Los resultados obtenidos en esta investigación muestran que entre más alto es el nivel escolar de las participantes menos creen en la maternidad como sentido de vida y como deber social; lo mismo ocurre con las mujeres que poseen un trabajo remunerado. Al parecer, a diferencia de las ideas que predominaban en generaciones pasadas, en la actualidad las mujeres consideran a la maternidad más que un rol social pre-establecido, un elemento que se suma a otros que dan sentido a su vida, pues proyectan el ser madres en función de intereses y planes a futuro, es decir, la maternidad ya no es vivida como algo que "tiene que ser" por mandato social, sino que es vista como un complemento de la vida femenina.

Los resultados indicaron que tanto las mujeres que sufrieron pérdida perinatal como aquellas que tuvieron problemas de infertilidad mostraron creencias más favorables hacia la maternidad en ambas subescalas, en comparación con las mujeres que ya tenían hijos y aquellas jóvenes que aún no los tenían. Estos resultados concuerdan con Molina (2006), que menciona que aun cuando el significado social de la maternidad tiene diferentes características dependiendo la clase social, la escolaridad, el momento histórico que se viva y la cultura en la que se está inmerso, el mandato social de "ser madres" todavía está presente en aquellas mujeres que, obligadas por determinadas causas de tipo médico, biológico o incluso social, no les es posible procrear.

Tales resultados también concuerdan con lo señalado por Nazario y Turato (2007), acerca de que, quienes no pueden concebir o parir, presentan un deseo más intenso por la maternidad, que puede estar provocado por la necesidad de confirmación del "adecuado" funcionamiento del organismo, así como por la exigencia del cumplimiento del rol social. En este sentido, la maternidad puede ser entendida por la mujer como una comprobación de su condición biológica, así como una obligación social en tanto que elige ser madre porque "debe" elegirlo, de acuerdo a un sistema de valores sociales que establece en qué momento se debe ejercer la maternidad. Este estudio mostró que la edad está relacionada con la valoración de la maternidad, ya que a medida que esta se incrementa, las creencias en la maternidad como sentido de vida se elevan.

Aunque no se puede negar la importancia de los aspectos biológicos involucrados en la maternidad, tampoco es posible dejar de lado el componente psicológico y social que gira en torno a ella, que muestra que la maternidad, más que instintiva o inherente al género femenino, es cultural y se va construyendo a lo largo de la historia según el contexto social en el que se vive.

Otros elementos importantes a considerar cuando se habla de las creencias que tienen las mujeres acerca de la maternidad son: la imagen que tienen de sí mismas, sus aspiraciones, deseos, proyectos de vida y de pareja; los cuales han ido modificando su ejercicio y han dado un sentido distinto a sus vidas.

No obstante, este estudio señala que para algunas mujeres la maternidad sigue estando idealizada y representa la parte central de su proyecto de vida personal, reafirmando su identidad como mujeres y obteniendo un estatus dentro de su medio social. Tal es el caso tanto de las participantes que no tienen hijos como de las que tienen más de dos, en las que se observaron creencias más favorables en torno a la maternidad como sentido de vida y como deber social, en comparación con aquellas mujeres que tuvieron de uno a dos hijos, las cuales reflejan puntajes más bajos en sus creencias.

Las mujeres que conformaron la muestra de este estudio pertenecen a zonas urbanas con acceso a servicios, educación, atención médica y posibilidades de empleo, elementos que pueden contribuir a que no focalicen la maternidad como fuente única de realización personal, por lo que se sugiere para futuras investigaciones aplicar la Escala de Creencias sobre la Maternidad a otras poblaciones de mujeres, como las procedentes de zonas rurales o de otras nacionalidades, e incluso a grupos de hombres, para obtener una visión más completa de las creencias, representaciones y comportamientos hacia la maternidad en diferentes contextos. 
Finalmente, se concluye que, más allá de los estereotipos socialmente establecidos, prevalece la idea de la maternidad como parte de la identidad femenina, basada en vivencias específicas que lleva a las mujeres a decidir tener o no hijos y realizarse en otras áreas de su vida. En suma, no existe una conducta universal para todas las madres. Los sentimientos, intereses y creencias respecto de los hijos y el ejercicio de la maternidad, varían dependiendo del contexto histórico, social y cultural, así como de las características individuales y psicológicas de cada mujer.

\section{Referencias}

Agudelo, L. J., Bedoya, G. J., \& Osorio, T. D. (2016). Ser mujer: Entre la maternidad y la identidad. Revista Poiésis, 306-313. doi: 10.21501/16920945.2121

Álvarez, B. (2013). La maternidad: Entre la decisión individual y/o la obligatoriedad social. En C. López, D. Marre, \& J. Bestard (Eds.) Maternidades, procreación y crianza en transformación (pp. 219-244). Barcelona, España: Ediciones Bellaterra

Arranz, L. L., Blum, G. B., Sauceda, G. L., \& Gutiérrez, A. R. (2001). Maternity desire in patients undergoing assisted reproduction treatment at a public health institution. Ginecologia y Obstetricia de México, 69, 5156.

Ávila, G. Y. (2005). Mujeres frente a los espejos de la maternidad: Las que eligen no ser madres. Desacatos, (17), 107-126.

Contreras, B. C., \& Reyes, L. I. (2007). Construcción y validación de una escala de control maternal. Revista Iberoamericana de Diagnóstico y Evaluación - e Avaliação Psicológica, 2(24), 9-21.

Casullo, M. (2009). La evaluación psicológica: modelos, técnicas y contextos. Revista Iberoamericana de Diagnóstico y Evaluación - e Avaliação Psicológica, 1(27), 9-28

Cárdenas, A. E., Chávez, S., \& Lozano, C. (2016). Actitudes de la pareja ante su infertilidad. Recuperado de http://bvirtual.ucol.mx/archivos/121_9706041 207.pdf
Colondro, C. L., Limiñana, G. R. M., \& Ordoñana, JR. (2014) Estilos de personalidad y adaptación psicológica en la toma de decisiones sobre lactancia en mujeres primíparas. Revista Iberoamericana de Diagnóstico y Evaluación - e Avaliação Psicológica, 2(38), 155-179.

Côté, A. D., \& Morrison, B. D. (2001). Women's voices reflecting changed expectations for pregnancy after perinatal loss. Journal of Nursing Scholarship, 33(3), 239-244. doi.org/10.1111/j.1547-5069.2001.00239.x

Ehrenberg, A. (2000). La fatiga de ser uno mismo, depresión y sociedad. Buenos Aires: Nueva visión.

Farkas-Klein, C. (2008). Escala de evaluación parental (EEP): Desarrollo, propiedades psicométricas y aplicaciones. Universitas Psychologica, 7(2), 457-467.

González, C. G., Carreño, M. J., Sánchez, B. C., \& Morales, C. FA. (2013) Estudio comparativo del autoconcepto en mujeres con esterilidad primaria y pérdida gestacional recurrente. Psicología y Salud, 19(2), 295-302

Maher, J. \& Saugeres, L. (2007). To be or not to be a mother? Women negotiating cultural representations of mothering. Journal of Sociology. $\quad 43(1), \quad$ 5-21. doi:10.1177/1440783307073931

Mendoza, H. (2012). La sexualidad reproductiva en el siglo XXI. El divorcio entre sexualidad y reproducción. Revista Perspectivas Sociales, 14(1), 33-46.

Mishtal, J. (2012). Irrational non-reproduction? The dying nation and the postsocialist logics of declining motherhood in Poland. Anthropology \& Medicine, 19(2), 153-169. doi:10.1080/13648470.2012.675048

Mitchell, D., \& Gray, E. (2007). Declining fertility: Intentions, attitudes and aspirations. Journal of Sociology, 43(1), 23-44. doi:10.1177/1440783307073933

Molina, M. E. (2006). Transformaciones histórico culturales del concepto de maternidad y sus repercusiones en la identidad de la mujer. Psykhe, 15(2), 93-103. doi:10.4067/S0718-22282006000200009

Mota G. C., Aldana C. E., \& Gómez L. M. E. (2013). Maternidad y pérdida perinatal: un 
duelo silencioso. Alemania: Editorial Académica Española.

Mota, G. C, Calleja, B. N., Gómez L. M. E., Aldana, C. E., \& Sánchez, P. M. A. (2010). Factores asociados con el duelo perinatal en mujeres con pérdida gestacional. Acta Psiquiatrica y Psicológica de América Latina. 56(4), 235-245

Nazario, R. D. C. P., \& Turato, E. R. (2007). Fantasías sobre embarazo y maternidad relatadas por mujeres adultas fértiles en hemodiálisis, sudeste de Brasil: Un estudio clínico-cualitativo. Revista Latino-americana de Enfermagem, 15(1), 55-61. doi:10.1590/S0104-11692007000100009

Prasopkittikun, T., Tilokskulchai, F., Sinsuksai, N., \& Sitthimongkol, Y. (2006). Self-efficacy in Infant Care Scale: Development and psychometric testing. Nursing and Health Sciences. 8, 44-50. doi:10.1111/j.1442-2018.2004.00266x

Rivero, N., Quintero, E., Montiel, C., Karame, A., \& Alarcón, A. (2015). Desajuste emocional y recursos adaptativos en parejas infértiles con tratamiento de reproducción asistida: Hallazgos preliminares. Recuperado de http://www.produccioncientificaluz.org/index. php/redieluz/article/viewFile/19727/19676

Romero, P. I., Sánchez, M. G., Romero, L. E., \& Chávez, C. M. (2010). Muerte materna: Una revisión de la literatura sobre factores socioculturales. Perinatología y Reproducción Humana, 24(1), 42-50.

Valladares, I.L. (1997). Revisión teórica sobre los mitos de la maternidad. Revista de Ciencias Sociales de la Universidad de Costa Rica, 65, 67-74 